Valéria Saraceni 1,2

Maria do Carmo Leal 2

\section{Avaliação da efetividade das campanhas para eliminação da sífilis congênita na redução da morbi-mortalidade perinatal. Município do Rio de Janeiro, 1999-2000}

\author{
Evaluation of the effectiveness of the congenital \\ syphilis elimination campaigns on reducing \\ the perinatal morbidity and mortality. \\ Rio de Janeiro, 1999-2000
}

1 Secretaria Municipal de Saúde do Rio de Janeiro. Rua Afonso Cavalcanti 455, Rio de Janeiro, $R J$ 20211-901, Brasil. 2 Departamento de Epidemiologia e Métodos Quantitativos em Saúde, Escola Nacional de Saúde Pública, Fundação Oswaldo Cruz. Rua Leopoldo Bulhões 1480, Rio de Janeiro, $R J$ 21041-210, Brasil. duca@ensp.fiocruz.br

\begin{abstract}
The growing incidence of congenital syphilis in Rio de Janeiro City led the City Health Department to organize the congenital syphilis Elimination Campaigns in 1999 and 2000, in order to call attention to the matter and to qualify health care personnel in the management of the disease. The aim of this paper was to evaluate the effectiveness of the campaigns, following the pregnant women identified with syphilis through the well-established health information systems (SINASC - Live births, SIM - mortality and SINAN - National disease surveillance data system). It became evident that a significant reduction on perinatal morbidity and mortality was achieved on the group of children born to mothers exposed to the campaigns, pointing out that the antenatal routine practice wasn't able to deal with the matter. The low number of congenital syphilis cases found on the surveillance system denotes a huge under-reporting of the disease.

Key words Congenital Syphilis; Sexually Transmitted Diseases; Health Campaigns; Perinatal Mortality
\end{abstract}

Resumo O aumento da incidência da sífilis congênita no Município do Rio de Janeiro levou a Secretaria Municipal de Saúde a realizar as campanhas para a sua eliminação nos anos de 1999 e 2000, com o objetivo de dar visibilidade à doença e capacitar os profissionais de saúde no manejo do agravo. Neste estudo realizou-se uma avaliação da efetividade das campanhas, servindo-se do seguimento das mulheres grávidas identificadas com sífilis durante as campanhas, pelos sistemas de informação em saúde (SINASC, SIM e SINAN) utilizando-se da comparação deste grupo a um grupo controle de gestantes não envolvidas na campanha. Foi possível evidenciar uma redução significativa da morbidade e mortalidade perinatais nos filhos das mulheres atendidas pelas campanhas, demonstrando que as ações da rotina de pré-natal nas unidades municipais não estavam conseguindo resolver o problema. Foi baixo o número de casos de sífilis congênita encontrado no SINAN em relação aos casos reconhecidos por intermédio das campanhas, demonstrando uma subnotificação importante.

Palavras-chave Sífilis Congênita; Doenças Sexualmente Transmissíveis; Campanhas de Saúde; Mortalidade Perinatal 


\section{Introdução}

A sífilis congênita é um agravo de saúde passível de eliminação, desde que a mulher infectada pelo Treponema pallidum seja identificada e tratada antes ou durante a gestação (Brown et al., 1970). Por ser uma doença de notificação compulsória, deve ser investigado e notificado todo caso de nascituro, vivo ou morto, filho de mãe com sífilis.

A sífilis é causa importante de morbidade e mortalidade perinatal. $\mathrm{O}$ tratamento da mulher grávida com penicilina benzatina, até trinta dias antes do parto, reduz, em muito, as complicações, como abortamento, prematuridade, natimortalidade, seqüelas nos bebês e óbito neonatal (Sanchez \& Wendel, 1997).

Com o objetivo de eliminação da sífilis congênita, o Ministério da Saúde (MS, 1993) recomenda o rastreio da sífilis na gravidez, utilizando-se o VDRL, teste quantitativo não treponêmico, na primeira consulta de pré-natal, no início do terceiro trimestre e na admissão para parto ou curetagem.

$\mathrm{O}$ aumento da incidência da sífilis congênita no Município do Rio de Janeiro a partir de 1996, levou a Secretaria Municipal de Saúde do Rio de Janeiro (SMS-RJ) a realizar as campanhas para sua eliminação nos anos de 1999 e 2000, visando a dar visibilidade à doença e treinar os profissionais de saúde para um atendimento pré-natal de maior qualidade, objetivando a redução da morbidade e da mortalidade perinatal (SMS-RJ, 1999).

As intervenções realizadas pelos serviços de saúde, embora muito freqüentes, de forma geral não são avaliadas quanto aos resultados alcançados, o que pode ter levado a uma sistemática repetição de equívocos, sem possibilidade de correção das práticas e melhoria efetiva das ações de saúde.

O conceito de avaliação que norteou este estudo diz que "avaliar é fazer um julgamento de valor a respeito de uma intervenção..." (Contandriopoulos et al., 1997:31). A pesquisa avaliativa, como descrita pelo mesmo autor, trata de um procedimento que faz um julgamento de uma intervenção usando métodos científicos. Deve-se verificar o contexto que gerou a intervenção e voltar seu olhar para este mesmo contexto após a mesma, estabelecendo como se deram as relações entre esses. Por tudo isso, deve ser capaz de gerar informações que colaborem para a tomada de decisões.

O conceito de efetividade varia com os autores e com o aspecto enfocado. Em muitos momentos, ele se confunde com o conceito de efi- cácia, eficiência e, até, qualidade, como colocam Silva \& Formigli (1994), discutindo os vários significados dessas palavras na literatura. Concordamos que a efetividade tem a ver com o "efeito das ações e práticas de saúde implementadas". A efetividade e o impacto estariam relacionados às modificações introduzidas por uma intervenção num contexto da vida real, que Contandriopoulos et al. (1997) chamam de eficácia de utilização.

O objetivo deste trabalho foi avaliar a efetividade das campanhas de eliminação da sífilis congênita, utilizando-se o seguimento das gestantes detectadas, usando os dados dos sistemas rotineiros de informação em saúde, o Sistema de Informações de Nascidos Vivos (SINASC), o Sistema de Notificação de Agravos (SINAN) e o Sistema de Informações de Mortalidade (SIM). Além disso, comparou-se as características das gestantes portadoras de sífilis e seus conceptos, com as gestantes não atendidas pelas campanhas e seus conceptos registrados nos mesmos sistemas de informação.

A hipótese do estudo foi a de que a realização de campanhas que envolvam o diagnóstico e tratamento da sífilis adquirida, na gestante durante o pré-natal, leva à diminuição da morbi-mortalidade perinatal por sífilis e, conseqüentemente, do número de casos de sífilis congênita.

\section{Métodos}

A população do estudo constituiu-se de gestantes com resultados de testes rápidos positivos para sífilis, obtidos durante as campanhas de eliminação da sífilis congênita, realizadas nas unidades municipais de saúde do Município do Rio de Janeiro com atendimento prénatal. Os dados foram coletados durante seis semanas consecutivas, nos meses de julho e agosto dos anos de 1999 e 2000 (Saraceni et al., 2000). As gestantes selecionadas para a realização de teste rápido para sífilis nas campanhas foram: as que não vinham recebendo assistência médica pré-natal; as que ainda não tinham realizado a primeira sorologia ou que já tinham e não sabiam do seu resultado e, por último, as gestantes que estavam no terceiro trimestre de gestação e que ainda não tinham realizado a segunda sorologia ou haviam feito e não tinham recebido seu resultado (SMS-RJ, 2000). No caso das mulheres já em acompanhamento pré-natal, a seleção para a realização ou não do teste rápido se deu por meio da consulta ao cartão da gestante e não do prontuário médico. $\mathrm{O}$ teste rápido utilizado (Determine $\mathrm{TP}$ - 
Abbott Laboratórios) é um teste bem mais caro em relação ao VDRL, de realização fácil, porém qualitativo, não permitindo o controle de cura sem a realização do VDRL.

Para cada gestante incluída na campanha foi preenchida uma ficha com dados de identificação e informações sobre a idade gestacional e assistência pré-natal. Esses dados foram coletados nas unidades de saúde, revisados e posteriormente codificados e digitados para análise.

Para a identificação das gestantes positivas das campanhas junto ao SINASC, ao SINAN e ao SIM (FUNASA, 1998), utilizou-se o programa RECLINK (Camargo Jr. \& Coeli, 1999), para o cruzamento inicial das informações. As gestantes não encontradas por este procedimento foram procuradas nominalmente.

Para cada ano foi obtida uma amostra aleatória dos dados do SINASC, utilizando-se o programa Epi Info versão 6.04, correspondendo à cerca de $1 \%$ dos registros gerais do SINASC para cada ano, a fim de elaborar o grupo-controle. As gestantes identificadas na campanha foram comparadas às das amostras em relação à idade materna, grau de instrução, município de residência, número de consultas de pré-natal, o local de ocorrência e tipo de parto. Para os recém-nascidos comparou-se o APGAR no quinto minuto (APGAR 5'), o peso ao nascer e a idade gestacional.

Com os dados do SINAN, as gestantes identificadas na campanha foram comparadas às demais com sífilis registradas no sistema quanto à idade materna; grau de instrução; município de residência; número de consultas de pré-natal; diagnóstico de sífilis durante a gravidez; realização de VDRL na primeira consulta, no terceiro trimestre e na hora do parto; história de natimortos nesta gestação e em gestações anteriores. Quanto ao concepto, comparou-se o peso ao nascer, idade gestacional, óbito neonatal, situação clínica do recém-nascido (assintomático ou não), realização de VDRL no sangue e líquor, e o resultado da radiografia de ossos longos.

Para a análise estatística foram utilizados testes qui-quadrado $\left(\chi^{2}\right)$ para testar hipóteses de homogeneidade entre proporções, comparando-se as variáveis relativas às gestantes, ao pré-natal, ao parto e nascimento e aos recémnatos das gestantes identificadas na campanha com as demais. O nível de significância foi estabelecido para um erro tipo I $(\alpha)$ de $5 \%$.

Todos os cuidados com a manutenção do sigilo dos dados foram tomados. Um único técnico trabalhou com as bases de dados que foram utilizadas. Em nenhum momento foram feitas citações nominais e os dados foram analisados agrupados.

\section{Resultados}

A campanha de eliminação da sífilis congênita do Município do Rio de Janeiro no ano de 1999, captou 9.428 gestantes, aproximadamente um quinto da clientela dos serviços de pré-natal das unidades de saúde municipais. No ano seguinte, o número de mulheres captadas foi de 7.413, registrando um decréscimo de $21,3 \%$.

Do conjunto das mulheres positivas para sífilis identificadas nas campanhas, $82,3 \%$ puderam ser identificadas no SINASC para o ano de 1999 e $67,7 \%$ para o ano de 2000 , constituindo-se em uma queda de $14,6 \%$.

Em relação ao SINAN, a perda foi consideravelmente maior do que no SINASC para os dois anos, mantido o padrão de uma pior cobertura no segundo ano. Em 1999, apenas 67 nascituros foram identificados entre os 475 esperados, ou seja, $14,1 \%$ do total. No ano seguinte, este porcentual foi de $10,4 \%$.

Mais grave ainda foi a subnotificação dos casos identificados em mulheres cujos partos ocorreram antes de trinta dias de instituído o tratamento para sífilis durante a campanha. Em 1999, 44 mulheres faziam parte deste grupo, e apenas 14 delas, $31,8 \%$, foram corretamente registradas no SINAN. Já em 2000, encontrou-se no SINAN apenas 7 das 33 mulheres, $21,2 \%$, na mesma situação.

O SIM, ao contrário dos sistemas anteriores, mostrou-se uma fonte fidedigna de informação, já que todos os natimortos e neomortos oriundos de mulheres que participaram das campanhas e identificados no SIM (seis casos) apresentaram como causa básica de óbito a sífilis congênita.

Na Tabela 1 foram comparadas as características das gestantes com sífilis identificadas nas campanhas e encontradas no SINASC, com as demais gestantes do SINASC. Decidiu-se analisar os anos de 1999 e 2000 juntos porque não houve diferença entre eles.

Não foram encontradas diferenças na composição etária entre as gestantes com sífilis das campanhas em relação ao conjunto das gestantes do SINASC. Quanto à escolaridade, esta foi significativamente mais baixa nas gestantes das campanhas, com $85,9 \%$ delas atingindo até o primeiro grau completo, contra $43,2 \%$ no SINASC. O parto cesáreo entre as mulheres das campanhas foi significativamente menor, $29,9 \%$, bem diferente do que se observa no SINASC, com 46,3\%. Para o APGAR 5', a prematu- 
Tabela 1

Comparação entre as gestantes com sífilis identificadas nas campanhas de eliminação da sífilis congênita e a amostra do SINASC. Município do Rio de Janeiro, Brasil, 1999 e 2000.

\begin{tabular}{|c|c|c|}
\hline & $\begin{array}{l}\text { Campanha } \\
(n=638)\end{array}$ & $\begin{array}{c}\text { SINASC } \\
(n=2.266)\end{array}$ \\
\hline \multicolumn{3}{|c|}{ Variáveis sócio-demográficas } \\
\hline \multicolumn{3}{|l|}{ Faixa etária (anos) } \\
\hline$<19$ & 20,1 & 21,3 \\
\hline $20-34$ & 68,7 & 67,6 \\
\hline$>34$ & 11,2 & 11,1 \\
\hline \multicolumn{3}{|l|}{ Nível de instrução* } \\
\hline 1ㅇ grau & 85,9 & 43,2 \\
\hline 2o grau & 11,4 & 33,1 \\
\hline$>2$ o grau & 2,7 & 23,7 \\
\hline \multicolumn{3}{|l|}{ Município de residência* } \\
\hline Rio de Janeiro & 97,0 & 90,1 \\
\hline Outros & 3,0 & 9,9 \\
\hline \multicolumn{3}{|l|}{$\begin{array}{l}\text { Variáveis relativas } \\
\text { ao parto e ao nascimento }\end{array}$} \\
\hline \multicolumn{3}{|l|}{$\begin{array}{l}\text { Número de consultas } \\
\text { de pré-natal* (SINASC) }\end{array}$} \\
\hline Nenhuma & 4,1 & 6,8 \\
\hline $1-3$ & 7,7 & 5,0 \\
\hline $4-6$ & 41,4 & 27,8 \\
\hline$>6$ & 46,8 & 60,4 \\
\hline \multicolumn{3}{|l|}{ Local de parto* } \\
\hline Municipal & 43,3 & 31,7 \\
\hline Estadual & 18,1 & 7,8 \\
\hline Federal + Universitária & 11,3 & 5,8 \\
\hline $\begin{array}{l}\text { Militar + Filantrópica + } \\
\text { Conveniada }\end{array}$ & 22,2 & 22,1 \\
\hline Privada & 5,1 & 32,6 \\
\hline \multicolumn{3}{|l|}{ Tipo de parto* } \\
\hline Vaginal & 70,1 & 53,7 \\
\hline Cesariana & 29,9 & 46,3 \\
\hline \multicolumn{3}{|l|}{ Apgar 5' } \\
\hline Até 7 & 3,9 & 4,2 \\
\hline$>7$ & 96,1 & 95,8 \\
\hline \multicolumn{3}{|l|}{ Peso ao nascer (gramas) } \\
\hline$<1.500$ & 2,7 & 1,6 \\
\hline $1.500-2.499$ & 8,0 & 7,8 \\
\hline $2.500 \mathrm{e}+$ & 89,3 & 90,6 \\
\hline \multicolumn{3}{|l|}{ Prematuridade } \\
\hline $\operatorname{Sim}$ & 8,0 & 7,0 \\
\hline Não & 92,0 & 93,0 \\
\hline
\end{tabular}

* p-valor $<0,05$

Fonte: Bancos de dados das campanhas de eliminação da sífilis congênita de 1999 e 2000, SINASC, 1999 e 2000, SMS-RJ. ridade e o baixo peso ao nascer (BPN), as proporções foram semelhantes entre o grupo de estudo e de comparação (Tabela 1).

Nas Tabelas 2 e 3, utilizando-se as informações do SINAN, comparou-se os casos confirmados de sífilis congênita das campanhas com os identificados pelo SINAN. Viu-se que as gestantes das campanhas não diferiram quanto à distribuição etária, mas apresentaram melhor nível de escolaridade e maior número de residentes no Município do Rio de Janeiro. Quanto à atenção pré-natal, 28,7\% das gestantes das campanhas fizeram sete e mais consultas contra $19,9 \%$ das outras. O diagnóstico da sífilis durante a gravidez também foi maior, $77,5 \%$ contra $52,1 \%$, bem como o percentual de positividade nos VDRL de primeira consulta e terceiro trimestre. Foi semelhante a proporção de natimortos anteriores nos dois grupos (Tabela 2).

Na Tabela 3 foi possível observar melhores resultados para os casos confirmados das campanhas. Foi mais baixa a proporção de mulheres com VDRL positivo no momento do parto $(89,8 \%$ vs. 96,9), menor a proporção de natimortos nesse grupo, $5,7 \%$ contra $8,3 \%$ do outro grupo, menor a de prematuros $(20,9 \%$ contra $25,2 \%)$ e de óbitos neonatais $(2,3 \%$ e $4,1 \%)$. Embora tenha se verificado uma redução do BPN no grupo das campanhas em comparação ao SINAN, $27,3 \%$ e $30,8 \%$, respectivamente, essa redução não foi significativa. A proporção de assintomáticos para sífilis congênita ao nascer foi a mesma nos dois grupos. Vê-se uma maior proporção de VDRL positivo no sangue periférico dos nascidos vivos das campanhas, em relação ao restante dos casos. A realização de VDRL no líquor e a radiologia de ossos longos apresentaram uma baixa proporção de realização. Entretanto, a positividade do VDRL no líquor e a presença de alteração radiológica foram significativamente menores entre os nascidos vivos das campanhas do que nos casos do SINAN.

Nas Tabelas 4 e 5, encontram-se os resultados referentes à análise em separado das mulheres que pariram antes de decorridos trinta dias do início do tratamento para sífilis.

Os casos do SINAN têm maior proporção de adolescentes, menor escolaridade e menor proporção de residentes no Município do Rio de Janeiro. As gestantes identificadas nas campanhas têm melhor acompanhamento pré-natal, maior diagnóstico da sífilis na gravidez e maior proporção de positividade ao VDRL na primeira consulta e menor proporção de natimortos anteriores.

Já na Tabela 5, que se refere aos recém-nascidos das gestantes considerados casos confir- 
mados, chama a atenção o fato de haver aqui uma maior proporção de natimortos entre os casos das campanhas, $19,0 \%$, contra $8,3 \%$ do SINAN e um mais alto percentual de BPN, 38,1\% vs. 30,6\%, ambos significativos. Contudo, a proporção de prematuridade foi a mesma nos grupos comparados.

A proporção de nascidos vivos sintomáticos para sífilis congênita ao nascimento foi alta, em coerência com demais achados desta tabela. Para cerca de dois terços dos recém-nascidos esta informação não foi preenchida, apesar de não necessitar de outros instrumentos além da avaliação clínica.

\section{Discussão}

Os dados deste estudo mostram que é possível avaliar os programas de saúde por intermédio dos Sistemas de Informação disponíveis, permitindo a aferição dos resultados alcançados pelas intervenções feitas.

No SINASC, sistema de informação que se atribui de boa qualidade e cobertura completa dos nascimentos vivos no Município do Rio de Janeiro (D'Orsi \& Carvalho, 1998; Silva et al., 1997), a perda das gestantes das campanhas poderia ter duas hipóteses explicativas. A primeira seria que uma parcela das mulheres que se encontravam fazendo pré-natal no Município do Rio de Janeiro residia e pariu fora, e a segunda seria a ocorrência de abortos. Como visto, foi baixo o percentual de não residentes que participaram da campanha, o que levaria a reforçar a segunda explicação. De fato, a distribuição da idade gestacional no momento da realização do teste para sífilis nas campanhas entre as gestantes não encontradas no SINASC, mostra que as perdas relativas às mulheres que se encontravam no primeiro trimestre de gestação foram bem maiores, $54,6 \%$, do que as do segundo e terceiro, $32,7 \%$ e $12,7 \%$. Neste último grupo, por definição, não se pode falar de um processo de abortamento.

Cerca de $15 \%$ do total das gestantes apresentam abortos espontâneos no primeiro trimestre da gravidez (Steer et al., 1989) e, para gestantes com sífilis, estima-se que as perdas sejam maiores. No presente estudo, as perdas de primeiro e segundo trimestres podem ser parcialmente atribuídas à sífilis, já que o aborto é um dos desfechos esperados. Na literatura encontram-se relatos de casos de abortos espontâneos, cujos laudos histopatológicos revelaram a presença do T. pallidum, tanto em tecido fetal como placentário, no primeiro e no segundo trimestres (Braunstein, 1978; Lee et
Tabela 2

Comparação entre as gestantes com sífilis identificadas nas campanhas de eliminação de sífilis congênita e as demais registradas no SINAN.

Município do Rio de Janeiro, Brasil, 1999 e 2000.

\begin{tabular}{|c|c|c|}
\hline & \multicolumn{2}{|c|}{ Confirmados } \\
\hline & $\begin{array}{c}\text { Campanha } \\
(\mathrm{n}=88)\end{array}$ & $\begin{array}{c}\text { SINAN } \\
(n=1917)\end{array}$ \\
\hline \multicolumn{3}{|c|}{ Variáveis sócio-demográficas } \\
\hline \multicolumn{3}{|l|}{ Faixa etária (anos) } \\
\hline$<20$ & 26,1 & 24,6 \\
\hline $20-34$ & 65,9 & 65,5 \\
\hline$>34$ & 8,0 & 9,9 \\
\hline \multicolumn{3}{|l|}{ Nível de instrução } \\
\hline 1으 grau & $85,0^{\star}$ & 91,5 \\
\hline 2o grau & 15,0 & 8,2 \\
\hline$>2$ o grau & 0,0 & 0,3 \\
\hline \multicolumn{3}{|c|}{ Município de residência } \\
\hline Rio de Janeiro & $98,9 *$ & 87,4 \\
\hline Outros & 1,1 & 12,6 \\
\hline \multicolumn{3}{|c|}{$\begin{array}{l}\text { Variáveis relativas } \\
\text { à assistência pré-natal }\end{array}$} \\
\hline \multicolumn{3}{|c|}{$\begin{array}{l}\text { Número de consultas } \\
\text { de pré-natal (SINAN) }\end{array}$} \\
\hline Nenhuma & 13,8 * & 29,1 \\
\hline $1-6$ & 57,5 & 51,0 \\
\hline$>6$ & 28,7 & 19,9 \\
\hline \multicolumn{3}{|l|}{ Sífilis na gravidez } \\
\hline Sim & $77,5^{\star}$ & 52,1 \\
\hline Não & 22,5 & 47,9 \\
\hline \multicolumn{3}{|l|}{ VDRL 1a consulta } \\
\hline Positivo & $79,7^{*}$ & 63,6 \\
\hline Negativo & 17,2 & 26,2 \\
\hline Não realizado & 3,1 & 10,2 \\
\hline \multicolumn{3}{|l|}{ VDRL 3ㅇ trimestre } \\
\hline Positivo & $72,1^{\star}$ & 55,5 \\
\hline Negativo & 23,3 & 20,0 \\
\hline Não realizado & 4,6 & 24,5 \\
\hline \multicolumn{3}{|c|}{ Natimortos anteriores } \\
\hline Não & 86,4 & 86,5 \\
\hline Sim & 13,6 & 13,5 \\
\hline
\end{tabular}

* $\mathrm{p}$-valor $<0,05$

Fonte: Bancos de dados das campanhas de eliminação da sífilis congênita de 1999 e 2000, SINASC, 1999 e 2000, SMS-RJ. 
Tabela 3

Comparação ente os nascituros com sífilis congênita identificados nas campanhas de eliminação de sífilis congênita e os demais registrados no SINAN. Município do Rio de Janeiro, Brasil, 1999 e 2000.

\begin{tabular}{|c|c|c|}
\hline & \multicolumn{2}{|c|}{ Confirmados } \\
\hline & $\begin{array}{l}\text { Campanha } \\
(\mathrm{n}=88)\end{array}$ & $\begin{array}{c}\text { SINAN } \\
(n=1.917)\end{array}$ \\
\hline \multicolumn{3}{|c|}{$\begin{array}{l}\text { Variáveis relativas ao parto } \\
\text { e ao nascimento }\end{array}$} \\
\hline \multicolumn{3}{|l|}{ VDRL parto } \\
\hline Positivo & $89,8^{*}$ & 96,9 \\
\hline Negativo & 10,2 & 2,0 \\
\hline Não realizado & 0,0 & 1,1 \\
\hline \multicolumn{3}{|l|}{ Natimorto } \\
\hline Sim & $5,7^{*}$ & 8,3 \\
\hline Não & 94,3 & 91,7 \\
\hline \multicolumn{3}{|c|}{ Peso ao nascer (gramas) } \\
\hline$<2.500$ & 27,3 & 30,8 \\
\hline $2.500 e+$ & 72,7 & 69,2 \\
\hline \multicolumn{3}{|l|}{ Prematuridade } \\
\hline Sim & $20,9^{*}$ & 25,2 \\
\hline Não & 79,1 & 74,8 \\
\hline \multicolumn{3}{|c|}{$\begin{array}{l}\text { Variáveis relativas } \\
\text { aos nascidos vivos }\end{array}$} \\
\hline \multicolumn{3}{|l|}{ Óbito } \\
\hline Sim & $2,3^{*}$ & 4,1 \\
\hline Não & 97,7 & 95,9 \\
\hline \multicolumn{3}{|l|}{ Assintomático } \\
\hline Sim & 73,7 & 71,1 \\
\hline Não & 26,3 & 28,9 \\
\hline \multicolumn{3}{|l|}{ VDRL sangue } \\
\hline Positivo & $79,0^{*}$ & 72,0 \\
\hline Negativo & 21,0 & 24,3 \\
\hline Não realizado & 0,0 & 3,7 \\
\hline \multicolumn{3}{|l|}{ VDRL líquor } \\
\hline Positivo & $2,3^{*}$ & 2,7 \\
\hline Negativo & 68,2 & 48,6 \\
\hline Não realizado & 29,5 & 48,7 \\
\hline \multicolumn{3}{|c|}{ Diagnóstico radiológico } \\
\hline Alterado & $2,2^{\star}$ & 5,8 \\
\hline Sem alteração & 97,8 & 94,2 \\
\hline
\end{tabular}

* p-valor $<0,05$.

Fonte: Bancos de dados das campanhas de eliminação da sífilis congênita de 1999 e 2000, SINASC, 1999 e 2000, SMS-RJ. al., 1994). Temmerman et al. (1992) demonstraram, em estudo caso-controle, uma associação entre aborto espontâneo e sorologia materna reativa para sífilis, com um risco 4,3 vezes maior de ocorrência de abortamento nas mulheres com sífilis, mesmo controlando para outros fatores. Resultados diferentes foram mostrados por Labbe et al. (2002) na GuinéBissau, em estudo caso-controle, demonstrando forte associação de sorologia positiva para sífilis com natimortalidade e parto prematuro, porém, não com abortamento espontâneo.

As perdas relativas ao SINAN retratam falhas na vigilância epidemiológica da sífilis congênita. Essas perdas, da ordem de 70 a $80 \%$, dimensionam a magnitude da subnotificação dos casos pelos estabelecimentos de saúde. Por outro lado, apontam para a necessidade de instituição dos serviços de epidemiologia hospitalares, que viessem a corrigir estas falhas. Atualmente, mais de $90 \%$ dos casos de sífilis congênita notificados no Município do Rio de Janeiro advêm das maternidades municipais (SMSRJ, 2000), cuja clientela não difere, do ponto de vista sócio-econômico, do restante da clientela das outras maternidades públicas do município. Das mulheres que participaram das campanhas, $43,3 \%$ delas foram atendidas nas maternidades municipais, proporção esta maior do que a sua representação no SINASC, que foi de $31,7 \%$. Quanto aos recém-nascidos que foram casos notificados de sífilis congênita, $92,4 \%$ deles nasceram em maternidades municipais, principais fontes de notificação. Esses dados refletem uma maior conscientização dessas maternidades em relação à vigilância epidemiológica da sífilis congênita, talvez em conseqüência dos diversos treinamentos dos profissionais de saúde já realizados nessas instituições que contam com a existência de serviços de epidemiologia hospitalar. Ainda assim, a notificação dos casos descartados parece não estar ocorrendo adequadamente, o que sinaliza para uma necessidade de reorientação do serviço de notificação.

Os problemas identificados no Município do Rio de Janeiro são semelhantes aos relatados em São Paulo por Tayra \& Matida (1997), que mostraram a dificuldade de investigação e notificação de casos de sífilis congênita e apontaram a sensibilização e capacitação dos profissionais como ferramentas estratégicas para conhecer a magnitude da incidência e, assim, almejar a eliminação desse agravo.

As falhas identificadas nos Sistemas de Informação refletem tanto um descuido com a notificação dos casos suspeitos de sífilis congênita pelos serviços de saúde, quanto proble- 
mas na qualidade da assistência ao pré-natal e ao parto.

Das campanhas de combate à sífilis congênita desenvolvidas pelo Município do Rio de Janeiro, a primeira, ocorrida no ano de 1999, teve maior adesão dos serviços de saúde do que a segunda. Isto pode ser denotado pela diminuição do número de serviços que participaram, caindo de 98 em 1999, para 95 em 2000, sendo que destes, 14 não aderiram por mais de três semanas. A desmobilização pode ser atribuída, em parte, à organização mais precária do segundo evento, que várias vezes teve seu início adiado por atraso na entrega dos kits diagnósticos.

Neste estudo, as mulheres diagnosticadas com sífilis durante a gestação corresponderam aos segmentos mais empobrecidos da população, evidenciado pela baixa escolaridade. Tal fato, corroborado pela literatura, foi mostrado por Behets et al. (1995) no Haiti, onde a positividade sorológica para sífilis esteve sempre associada ao analfabetismo e baixo nível sócioeconômico.

Quanto ao acompanhamento pré-natal, as gestantes da campanha se apresentaram em situação de desvantagem quando considerado o número mínimo adequado de consultas, mas não apresentaram diferenças em relação ao conjunto de gestantes do Município do Rio de Janeiro em relação às condições de nascimento da criança.

Apesar da pior situação social e das perdas de informações relatadas, as campanhas contribuíram para que essas mulheres tivessem maior chance de diagnóstico da sífilis durante a gestação. Dentre todos os casos confirmados e mesmo nas que tiveram o diagnóstico próximo ao parto, foi maior o porcentual de identificação prévia da doença. Da mesma forma, a positividade do VDRL no momento do parto foi significativamente menor no grupo das campanhas, refletindo a eficácia do tratamento.

Os benefícios das campanhas se estenderam também para a redução da morbidade e da mortalidade relacionadas à sífilis nos recémnascidos. As reduções dos índices de prematuridade, natimortalidade e neomortalidade foram significativas no grupo captado. Embora tenha havido também diminuição no BPN, as diferenças entre os dois grupos não alcançaram significância estatística.

No grupo com intervalo menor do que trinta dias entre o diagnóstico e o parto verificouse que os óbitos neonatais foram evitados. Contudo, o diagnóstico e tratamento tardio não diminuíram a natimortalidade e mantiveram o retardo de crescimento intra-uterino, aqui de-
Tabela 4

Comparação entre as gestantes com sífilis, identificadas nas campanhas de eliminação de sífilis congênita, cujos partos ocorreram antes de decorridos 30 dias do início do tratamento para sífilis, e as demais registrados no SINAN. Município do Rio de Janeiro, Brasil, 1999 e 2000.

\begin{tabular}{|c|c|c|}
\hline & \multicolumn{2}{|c|}{ Confirmados } \\
\hline & $\begin{array}{l}\text { Campanha } \\
\qquad(n=21)\end{array}$ & $\begin{array}{c}\text { SINAN } \\
(n=1.984)\end{array}$ \\
\hline \multicolumn{3}{|c|}{ Variáveis sócio-demográficas } \\
\hline \multicolumn{3}{|c|}{ Faixa etária (anos)* } \\
\hline$<20$ & 14,3 & 24,6 \\
\hline $20-34$ & 71,4 & 65,5 \\
\hline$>34$ & 14,3 & 9,9 \\
\hline \multicolumn{3}{|c|}{ Nível de instrução* } \\
\hline 1ㅇ grau & 63,2 & 91,5 \\
\hline 2o grau & 31,6 & 8,3 \\
\hline$>2$ o grau & 5,2 & 0,2 \\
\hline \multicolumn{3}{|c|}{ Município de residência* } \\
\hline Rio de Janeiro & 95,2 & 87,7 \\
\hline Outros & 4,8 & 12,3 \\
\hline \multicolumn{3}{|c|}{$\begin{array}{l}\text { Variáveis relativas à } \\
\text { assistência pré-natal }\end{array}$} \\
\hline \multicolumn{3}{|c|}{$\begin{array}{l}\text { Número de consultas } \\
\text { de pré-natal (SINAN)* }\end{array}$} \\
\hline Nenhuma & 14,3 & 28,7 \\
\hline $1-6$ & 61,9 & 51,1 \\
\hline$>6$ & 23,8 & 20,2 \\
\hline \multicolumn{3}{|c|}{ Sífilis na gravidez* } \\
\hline Sim & 66,7 & 53,2 \\
\hline Não & 33,3 & 46,8 \\
\hline \multicolumn{3}{|l|}{ VDRL 1a consulta* } \\
\hline Positivo & 80,0 & 64,5 \\
\hline Negativo & 20,0 & 25,6 \\
\hline Não realizado & 0,0 & 9,9 \\
\hline \multicolumn{3}{|c|}{ VDRL 3으 trimestre* } \\
\hline Positivo & 85,7 & 56,0 \\
\hline Negativo & 14,3 & 18,5 \\
\hline Não realizado & 0,0 & 25,5 \\
\hline \multicolumn{3}{|c|}{ Natimortos anteriores* } \\
\hline Não & 76,2 & 86,6 \\
\hline Sim & 23,8 & 13,4 \\
\hline
\end{tabular}

* p-valor < 0,05;

Fonte: Bancos de dados das campanhas de eliminação da sífilis congênita de 1999 e 2000, SINASC, 1999 e 2000, SMS-RJ. 
Tabela 5

Comparação entre os nascituros com sífilis congênita, identificados nas campanhas de eliminação da sífilis congênita, cujos nascimentos ocorreram antes de decorridos 30 dias de instituído o tratamento materno para sífilis, e os demais registrados no SINAN. Município do Rio de Janeiro, Brasil, 1999 e 2000.

\begin{tabular}{|c|c|c|}
\hline & \multicolumn{2}{|c|}{ Confirmados } \\
\hline & $\begin{array}{c}\text { Campanha } \\
(n=21)\end{array}$ & $\begin{array}{c}\text { SINAN } \\
(n=1.984)\end{array}$ \\
\hline \multicolumn{3}{|c|}{$\begin{array}{l}\text { Variáveis relativas ao parto } \\
\text { e ao nascimento }\end{array}$} \\
\hline \multicolumn{3}{|l|}{ VDRL no parto* } \\
\hline Positivo & 100,0 & 96,8 \\
\hline Negativo & 0,0 & 2,1 \\
\hline Não realizado & 0,0 & 1,1 \\
\hline \multicolumn{3}{|l|}{ Natimorto* } \\
\hline Sim & 19,0 & 8,2 \\
\hline Não & 81,0 & 91,8 \\
\hline \multicolumn{3}{|c|}{ Peso ao nascer (gramas)* } \\
\hline$<2.500$ & 38,1 & 30,6 \\
\hline $2.500 \mathrm{e}+$ & 61,9 & 69,4 \\
\hline \multicolumn{3}{|l|}{ Prematuridade } \\
\hline Sim & 22,2 & 23,2 \\
\hline Não & 77,8 & 76,8 \\
\hline \multicolumn{3}{|c|}{$\begin{array}{l}\text { Variáveis relativas } \\
\text { aos nascidos vivos }\end{array}$} \\
\hline \multicolumn{3}{|l|}{ Óbito* } \\
\hline Sim & 0,0 & 4,1 \\
\hline Não & 100,0 & 95,9 \\
\hline \multicolumn{3}{|l|}{ Assintomático* } \\
\hline Sim & 57,1 & 71,3 \\
\hline Não & 42,9 & 28,7 \\
\hline \multicolumn{3}{|l|}{ VDRL sangue* } \\
\hline Positivo & 76,5 & 72,3 \\
\hline Negativo & 23,5 & 24,2 \\
\hline Não realizado & 0,0 & 3,5 \\
\hline
\end{tabular}

* p-valor $<0,05$.

Fonte: Bancos de dados das campanhas de eliminação da sífilis congênita de 1999 e 2000, SINASC, 1999 e 2000, SMS-RJ. monstrado pelo maior índice de BPN. A proporção de recém-nascidos sintomáticos foi bem maior neste grupo, confirmando que o tratamento tardio não reduz a morbidade relacionada à sífilis na gestação.

A análise mais refinada da morbidade do recém-nascido referente à possibilidade de neurossífilis ou lesões ósseas ficou muito prejudicada pelo grande número de fichas com informações ignoradas, exames não realizados ou caselas não preenchidas.

\section{Conclusão}

As campanhas para eliminação da sífilis congênita associaram-se a uma redução da morbi-mortalidade perinatal, como pode ser evidenciado pela diminuição significativa da natimortalidade, da neomortalidade e da prematuridade. Entretanto, nos casos tratados tardiamente não houve o mesmo benefício, sendo, aparentemente, evitada apenas a neomortalidade.

As campanhas vieram chamar a atenção dos profissionais de que os atos da rotina de pré-natal não estavam resolvendo o problema da sífilis congênita. As diferenças significativas encontradas nas gestantes que sofreram a intervenção das campanhas corroboram esse fato. Pode-se dizer que as campanhas tiveram efetividade para modificar a situação da sífilis congênita no Município do Rio de Janeiro e que os resultados alcançados aqui reafirmam as campanhas como instrumentos de política pública de saúde, possíveis agentes transformadores da situação atual da sífilis congênita.

O SINAN precisa ser revisto e sua qualidade verificada por meio de um estudo de validação, que oriente as futuras ações de vigilância epidemiológica. 


\section{Referências}

BEHETS, F. M.; DESORMEAUX, J.; JOSEPH, D.; ADRIEN, M.; COICOU, G.; DALLABETA, G.; HAMILTON, H. A.; MOENG, S.; DAVIS, H. \& COHENS, M. S., 1995. Control of sexually transmitted diseases in Haiti: Results and implications of a baseline study among pregnant women living in City Soleil Shatytowns. Journal of Infectious Diseases, 172:764-771.

BRAUNSTEIN, H., 1978. Congenital syphilis in aborted second trimester fetus: Diagnosis by histological study. Journal of Clinical Pathology, 31:265267.

BROWN, W. J.; DONOHUE, J. F.; AXNICK, N. W.; BLOUNT, J. H.; EWE, N. H. \& JONES, O. J., 1970. Syphilis and Other Venereal Diseases. Cambridge: Harvard University Press.

CONTANDRIOPOULOS, A. P.; CHAMPAGNE, F.; DENIS, J. L. \& PINEAULT, R., 1997. A avaliação na área da saúde: Conceitos e métodos. In: Avaliação em Saúde: Dos Modelos Conceituais à Prática na Análise da Implantação de Programas (Z. M. A. Hartz, org.), pp. 29-48, Rio de Janeiro: Editora Fiocruz.

D'ORSI, E. \& CARVALHO, M. S., 1998. Perfil de nascimentos no Município do Rio de Janeiro: Uma análise espacial. Cadernos de Saúde Pública, 14: 367-379.

CAMARGO Jr., K. R. \& COELI, C. M., 1999. Software RECLINK, Relacionamento Probabilístico de Registros. Rio de Janeiro: Universidade do Estado do Rio de Janeiro.

FUNASA (Fundação Nacional de Saúde), 1998. Guia de Vigilância Epidemiológica. 5a Ed. Brasília: FUNASA/Ministério da Saúde.

LABBE, A. C.; MENDONÇA, A. P.; ALVES, A. C.; JAFFAR, S.; DIAS, F.; ALVARENGA, I. C.; FROST, E.; MORENCY, P.; MILORD, F. \& PEPIN, J., 2002. The impact of syphilis, HIV-1 and HIV-2 on pregnancy outcome in Bissau, Guiné-Bissau. Sexually Transmissible Diseases, 29:157-167.

LEE, W. K.; SCHWARTZ, D. A.; RICE, R. J. \& LARSEN, S. A., 1994. Syphilitic endometritis causing first trimester abortion: A potential infectious cause of fetal morbidity in early gestation. Southern Medical Journal, 87:1259-1261.
MS (Ministério da Saúde), 1993. Bases Técnicas para a Eliminação da Sífilis Congênita. Brasília: Coordenação Nacional de DST/AIDS, Secretaria de Políticas de Saúde, MS.

SANCHEZ, P. J. \& WENDEL, G. D., 1997. Syphilis in pregnancy. Clinics in Perinatology, 24:71-90.

SARACENI, V.; NICOLAI, C.; DOMINGUES, R.; LAURIA, L. M.; DUROVNI, B. \& BOARETTO, M. C., 2000. Sífilis adquirida e sífilis congênita: Ações do Município do Rio de Janeiro. DST - Jornal Brasileiro de Doenças Sexualmente Transmissíveis, 12:95.

SILVA, L. M. V. \& FORMIGLI, V. L. A., 1994. Avaliação em saúde: Limites e perspectivas. Cadernos de Saúde Pública, 10:80-91.

SILVA, R. I.; THEME FILHA, M. M. \& NORONHA, C. P., 1997. Sistema de informações sobre nascidos vivos na cidade do Rio de Janeiro. Informe Epidemiológico do SUS, 6:33-48.

SMS-RJ (Secretaria Municipal de Saúde do Rio de Janeiro), 1999. Sífilis na gravidez. Saúde em FocoDados, 23:2-7.

SMS-RJ (Secretaria Municipal de Saúde do Rio de Janeiro), 2000. Sífilis na gravidez. Saúde em FocoDados, 24:13-16.

STEER, C.; CAMPBELL, S.; DAVIES, M.; MASON, B. \& COLLINS, W. P., 1989. Spontaneous abortion rates after natural and assisted conception. BMJ, 299: 1317-1318.

TAYRA, A. \& MATIDA, L. H., 1997. Relatório dos casos identificados de sífilis congênita: 1995. DST - Jornal Brasileiro de Doenças Sexualmente Transmissíveis, 9:24-29.

TEMMERMAN, M.; LOPITA, M. I.; SANGHVI, H. C.; SINEI, S. K.; PLUMMER, F. A. \& PIOT, P., 1992. The role of maternal syphilis, gonorrhoea and HIV-1 infections in spontaneous abortion. International Journal of STD and AIDS, 3:418-422.

Recebido em 5 de novembro de 2002

Versão final reapresentada em 27 de fevereiro de 2003

Aprovado em 8 de maio de 2003 